\title{
Late-emerging strains of HIV induce T-cell homeostasis failure by promoting bystander cell death and immune exhaustion in naïve CD4 and all CD8 T-cells
}

\author{
Catherine N. Kibirige*, Frederick A. Menendez, Hao Zhang, Tricia L. Nilles, Susan Langan, \\ Joseph B. Margolick \\ Department of Molecular Microbiology and Immunology, Johns Hopkins Bloomberg School of Public Health, Baltimore, MD 21205, United States
}

\section{A R T I C L E I N F O}

\section{Article history:}

Received 13 June 2013

Accepted 1 April 2014

\begin{abstract}
A B S T R A C T
The mechanisms involved in the decline of CD4 and CD8 T-cells that lead to HIV-induced immune dysregulation are not clearly understood. We hypothesize that late-emerging strains of HIV, such as CXCR4tropic (X4) virions, induce T-cell homeostasis failure by promoting significantly more bystander cell death, and immune exhaustion in naïve CD4 and all CD8 T-cells, when compared to strain of HIV, such as CCR5-tropic (R5) virions, found early during the course of infection. In the reported study, inactivated $\mathrm{X} 4$ virions induced greater bystander cell death in sort-purified naïve CD4 T-cells compared to R5 virions, which was significant $(p=0.013)$, and in memory CD8 T-cells, though the latter was not significant. A clearer understanding of the mechanisms involved in HIV-induced depletion of T-cell numbers and function could lead to therapies that prevent T-cell death and restore immune function. These therapies could improve current anti-retroviral and cure-related treatments by boosting the immune system's own ability to combat the virus.
\end{abstract}

(c) 2014 Elsevier Ltd. All rights reserved.

\section{Introduction}

Despite many years of intense research, the precise mechanisms that lead to the development of AIDS after infection with HIV are not well defined. Effective therapeutic strategies that enhance the immune system's ability to combat the disease over prolonged periods of infection can only be devised when a clearer understanding of these mechanisms is obtained.

One hallmark of HIV-1 infection is the gradual loss of CD4 T-cells over time. This loss begins with the establishment of HIV-1 infection and is initially counterbalanced by an increase in CD8 T-cells (except in individuals with very rapid disease progression). This allows for the maintenance of a constant level of total circulating CD3 T-cells, despite an inversion in the CD4/CD8 ratio, for many years, a phenomenon known as blind T-cell homeostasis (TCH) [1-8]. In the vast

Presented in part as poster "LB-24" at the 47th annual meeting of the Infectious Diseases Society of America in Philadelphia, PA Oct 29-Nov 12009.

Funding was received from the Fogarty International Center/USNIH: Grant \# 2 D 43 TW000010-16 - AITRP, and NIH grants U01-AI-35042 and M01-RR-00052(GCRC).

* Corresponding author. Address: Johns Hopkins University, Bloomberg School of Public Health, Department of Molecular Microbiology and Immunology, $615 \mathrm{~N}$ Wolfe Street, Baltimore, MD 21205, United States. Tel.: +1 410955 4825; fax: +1 4109550105.

E-mail address: ckibiri1@jhu.edu (C.N. Kibirige). majority of cases, HIV-1 infection, if not treated, leads to AIDS, with $\mathrm{TCH}$ failure (i.e., the loss of both CD4 and CD8 T-cells) occurring an average of $1.5-2.5$ years before clinically-defined AIDS [5,6,8]. The time between the establishment of HIV-1 infection and TCH failure is thus more variable than the time between $\mathrm{TCH}$ failure and the onset of clinically-defined AIDS. This suggests a common mechanism of disease progression between $\mathrm{TCH}$ failure and the development of AIDS. The emergence of variants of HIV that use CXCR4 as a co-receptor, which has long been associated with accelerated progression of HIV disease [9-11], most commonly occurs in the year immediately preceding TCH failure $[6,12,13]$.

Naïve T-cells are distinguished by the expression of high levels of the CXCR4 receptor. They are considered critical for the replenishment of the immune system after an infection because they are long-lived and have the capacity to proliferate greatly and differentiate into memory and effector T-cells. The emergence of X4 virions coincides with accelerated CD4 T-cell decline and with the onset of overall CD8 T-cell decline. Naïve CD8 T-cell levels, in particular, have been shown to decline steadily throughout the course of HIV disease [14]. Despite controversy, there are an increasing number of reports in the literature that HIV can actually infect CD8 Tcells [15], including naïve CD8 T-cells [16].

Our data, along with other findings in the literature, suggest that late-emerging strains of HIV, such as X4 strains, may actively 
target naïve CD4 T-cells in particular and also directly affect CD8 Tcells overall. These events could be the key factors that tip the balance into the severe immune dysregulation that leads to AIDS.

\section{Hypothesis}

We hypothesize that, non-infectious virions, derived from lateemerging X4 and highly pathogenic R5 virions, contribute to T-cell homeostasis failure during HIV disease progression by depleting uninfected, naïve, CXCR4-positive, CD4 T-cells and affecting the viability and survival of CD8 T-cells overall. This hypothesis could help us understand the bystander immunological effects of HIV ligand binding and direct research towards new therapeutic strategies to inhibit these effects.

\section{Hypothesis evaluation}

HIV-induced bystander cell death by non-infectious mechanisms

Only a very small proportion of circulating T-cells are found to be infected with HIV at any one time. Most of the cell death that occurs is in uninfected, "bystander" T-cells [3,13,17-22]. Additionally, the vast majority of circulating HIV virions in vivo are defective and non-infectious [3,12,20,22-26]. They are, however, capable of triggering $\mathrm{T}$-cell death and stimulating partial immune activation through interaction with surface receptors on T-cells $[27,28]$ even without complete cell infection.

To verify this, we studied the survival of CD4 and CD8 T-cells after exposure to primary strains of HIV that had been inactivated with 2,2'-dithiodipyridine (aldrithiol). Aldrithiol covalently modifies essential zinc fingers in the HIV nucleocapsid protein and arrests HIV infectivity at the reverse transcription step $[7,13]$. Unlike other methods, such as exposure to heat or formalin, this method preserves the conformational and functional integrity of virion surface proteins so that virions can undergo cognate interactions with CD4 and perhaps CCR5 and CXCR4 [13,28,29]. These aldrithiol-inactivated virions have been shown to interact with T-cells without leading to active infection [28].

The emergence of highly pathogenic strains of HIV as a trigger for Tcell homeostasis failure

Variants of HIV that utilize the CXCR4 coreceptor have long been associated with accelerated disease progression [9-11]. The emergence of X4 variants most commonly occurs in the year immediately preceding $\mathrm{TCH}$ failure $[6,12,13]$. In longitudinal studies of Clade B HIV-1 infection, the average CD4 T-cell count at which X4 viruses are first detected is approximately 440 cells $/ \mu \mathrm{l}$ $[10,30]$, while that at the time of T-cell homeostasis failure is approximately 350 cells $/ \mu 1$ [31], suggesting that the emergence of $\mathrm{X} 4$ viruses precedes TCH failure.

CXCR4 tropic strains of HIV have been shown to emerge in $50 \%$ or more of HIV infected people [32]. Discrepancies in the literature about the extent of $\mathrm{X} 4$ switching exist because assays and algorithms used to determine viral tropism differ considerably [33]. Despite these discrepancies, studying differences in the effect of CXCR4-tropic as compared to CCR5-tropic virions on the immune system should provide key insights into the pathogenic mechanisms of HIV.

The loss of naïve T-cells as a major determinant of AIDS development

A stable pool of naïve T-cells is ordinarily maintained by the thymus via the release of immature lymphocytes [34] and by the proliferation of peripheral naïve cells. Lymphocytes released by the thymus serve as peripheral precursor cells for regenerating mature naïve T-cells. Naïve T-cells have a major role in maintaining T-cell diversity, replenishing effector/memory T-cell populations and protecting the integrity of the total T-cell pool [34]. The disruption of CD4 and eventually CD8 T-cell homeostasis occurs most prominently in the naïve, CXCR4+ T-cell compartment. It is therefore conceivable that a key switch in the development of AIDS could occur when strains of HIV emerge that are more capable of targeting this particular subset of T-cells. The rhesus macaque animal model for HIV-1 pathogenesis corroborates this hypothesis in that some highly pathogenic strains of SHIV exclusively use CXCR4 for cell entry and target naïve CD4+ T-cells for infection. This is in contrast to natural SIV infection in macaques, which utilizes CCR5 and targets memory and effector CD4 T-cells.

Loss in CD8 T-cell numbers and function as a major determinant of AIDS development

CD4 T-cells are the main cells affected by HIV, because most viral replication takes place in them and their decline is the most clinically important feature of HIV infection. CD8 T-cells are also important in HIV infection, however, because they are essential for viral control and host survival through direct killing of infected cells and secretion of factors such as cytotoxic T-cell associated response factor (CNAR), which suppresses HIV replication noncytolytically $[32,35]$. Their importance can be seen in that HIV-specific CD8 T-cells increase concurrently with the decline in viral load shortly after acute HIV infection. Experiments in animal models corroborate this in that depletion of CD8+ T-cells causes high viral loads and rapid disease progression in SIV infected monkeys [36,37]. Additionally, CD8 T-cells provide help to CD4 T-cells by secreting IL-2 and other cytokines required for the maintenance of their numbers and function [38].

Effective control of HIV infection is associated with polyfunctional CD8+ T-cells that are more capable of degranulation and producing multiple functional molecules, such as interleukin 2 (IL-2), interferon-gamma (IFN $\gamma$ ) and tumor necrosis factor (TNF) [35]. HIV-specific CD8+ T-cells from HIV-infected progressors, for example, proliferate poorly and secrete fewer cytokines and chemokines [39]. The up-regulation of the programmed cell death molecule 1 (PD-1) and its ligand PD-L1, on both CD4 and CD8 T-cells is associated with decreased T-cell proliferation and cytokine production and poor T-cell survival [40]. This was corroborated in the present study in that up regulation of PD- 1 was found in naive CD8 T-cells exposed to the aldrithiol-inactivated virions. For these reasons, it is important that we improve our understanding of the factors that affect CD8+ T-cell numbers and function during HIV disease progression.

HIV surface glycoproteins have a well-characterized specificity for the CD4 molecule and are not generally thought to interact directly with CD8 T-cells. How then might HIV virions affect CD8 T-cell numbers and function? There are several theories that could explain this. CD8 T-cell precursors within the thymus are CD4/CD8 double positive and are CXCR4-positive which presents an opportunity for interaction with HIV viral particles via the CD4 and CXCR4 receptors $[16,41,42]$. Indeed, HIV has been shown to infect and deplete $\mathrm{CD} 4 / \mathrm{CD} 8$ double positive thymocytes [43]. Additionally, activated effector and memory CD8 T-cells have been shown to re-express the CD4 molecule [44,45], which could allow them to interact with HIV virions via this receptor. It has also been shown that HIV can infect CXCR4 positive cells in vitro in the absence of the CD4 molecule [46]. It is therefore conceivable that late-emerging strains of HIV, such as X4 virions, interact directly with CD8 T-cells via the CXCR4 receptor, depleting their numbers and causing a loss in their function in a more profound manner than the strains present earlier during infection. 
Empirical data

See Table 1.

\section{Consequences of the hypothesis and discussion}

The most significant finding in the reported study was that sortpurified naïve CD4 T-cells exposed to inactivated X4 virions underwent significantly more cell death than those exposed to R5 virions (Table 1). It was also interesting that memory CD8 T-cells showed a greater decline, albeit statistically insignificant, when exposed to inactivated X4 virions, as compared to R5 virions. These results support previously published data showing that inactivated virions can in fact induce bystander T-cell death [28], and extend these findings by showing that this death can be induced differentially by different strains of HIV, among unstimulated T-cell subsets. These in vitro findings are also consistent with previous in vivo studies showing that naïve T-cells decline significantly in number around the T-cell inflection point [27] and with the hypothesis of this study that late-emerging strains of HIV contribute to T-cell homeostasis failure by inducing more cell death in naïve CD4 Tcells than the R5 strains present earlier during HIV infection. The findings also lend support to the hypothesis that late-emerging strains of HIV have a more deleterious effect on CD8 T-cells when compared to viral strains found earlier during the course of infection.

Data from the MACS and other studies show that the mean CD4 T-cell count at the time of TCH failure is about 350 cells/ $\mu \mathrm{l}[6,10]$ and that after $\mathrm{TCH}$ failure CD4 T-cell counts fall to approximately 200 cells/ $\mu$ l over approximately 2 years, representing an accelerated cellular decline of 0.21 cells/ $\mu \mathrm{l} /$ day $[6,49]$. In Table 1 , the difference calculated for the decrease in viable naïve CD4 T-cells induced by $\mathrm{X} 4$ virions as compared to $\mathrm{R} 5$ virions was $0.09 \pm 0.04$ cells $/ \mu \mathrm{l} / \mathrm{day}$. This is close to the value estimated in the above-mentioned studies, allowing for the influence of in vivo factors other than the virus itself that may augment the decline in CD4 T-cells.

It was originally believed that HIV required CD4 T-cells to be activated in order to infect or stimulate them [50]. In our study and in a study by Esser and colleagues, T-cells were exposed to aldrithiol-inactivated HIV virions without any exogenous stimulation. The cells became partially activated, CD95+CD95L+CD69-CD25-, as opposed to fully activated CD95+CD95L+CD69+CD25+, before undergoing programmed cell death [28] which suggests that complete activation of naïve T-cells is not required for the induction of bystander T-cell death. Additionally, the present study found that naïve CD8 T-cells exposed to the inactivated virions up-regulated PD-1, a marker for cellular activation and T-cell exhaustion, and down-regulated CD31 (data not shown). High expression of CD31 (CD31 ${ }^{\text {bright }}$ ) characterizes T-cells that have not undergone any activation or proliferation [51]. The down-regulation of CD31 is consistent with a "partially activated" phenotype.

\section{The development of HIV immune-restorative therapies}

In the light of these hypotheses, future studies into HIV immune-restorative, therapeutic vaccine or cure-related therapies should focus more on preventing cell death in conjunction with preventing T-cell exhaustion and restoring immune function. Treatment evaluation should be done in both CD4 and CD8 T-cells. Future studies should focus more on bystander mechanisms of immune dysregulation and how ligand binding by viral particles, even in a non-infectious manner, affects immune status. Studies should be designed to evaluate whether possible immune therapies prevent highly pathogenic strains of HIV from exerting their damaging effects when compared to less pathogenic HIV variants. Studies should determine whether immune therapies specifically arrest or reverse T-cell homeostasis failure based on more extensively defined biomarkers.

IL-15, IL-7, IFN $\alpha$, IL-21, anti-TNFRII, PD-1/PD-L1 blockers, gp41 fusion inhibitors, HIV Env surface glycoprotein binding agents and IL-2, for example, have all been suggested as possible immune-therapies against AIDS development. Specifically, IL-15 and IFN $\alpha$ have been shown to restore CNAR activity in a number of studies [32]. IL-15 has also been found to have anti-apoptotic effects in CD8 Tcells [52]. The in vivo secretion of IL-21 by HIV-specific CD8+ T-cells from elite controllers has been linked with the maintenance of CD8 T-cell levels and secretion of IL-2 [39]. Culture and human studies show that CD8+ T-cell apoptosis can be inhibited by antibodies against TNFRII [53]. PD-1 and PD-L1 blockers have been shown to be effective at reviving CD8 effector function during HIV infection [54]. HIV gp41 fusion inhibitors such as T20 and C34 have been shown to inhibit bystander cell death in vitro [55]. HIV envelope glycoprotein (Env) binding agents such as plant lectins and glycopeptide antibodies could potentially prevent the bystander effects cause by HIV particles and soluble gp120 [23].

IL-2 therapy, as a final example, has been shown to cause an early increase in CD45RO+ effector memory CD4 T-cells, followed by a rise in CD45RA+ naïve and central memory CD4 T-cells [38]. It has also been shown to restore CNAR activity in CD8 T-cells in a number of studies [32]. When combined with HAART, IL-2 immunotherapy has been shown to increase HIV-1 specific CD8+ T-cell responses in infected subjects. In 2010, Margolick et al. entered a 29 year old, HIV+ male patient into an Ultra Low Dose (UDL) IL-2 therapy study with 2 ART (zidovudine, lamivudine and efavirenz) interruptions, with relative success. Viral load had been undetect-

Table 1

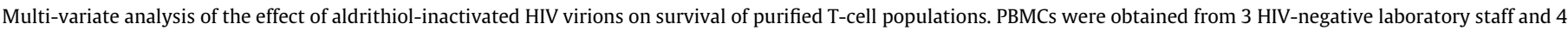

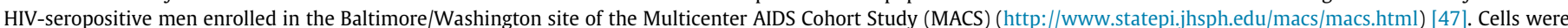

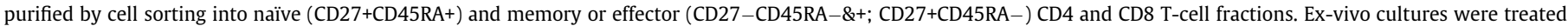

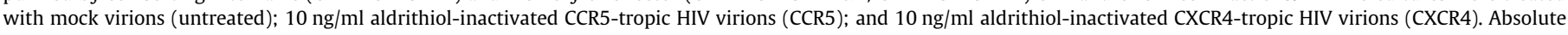

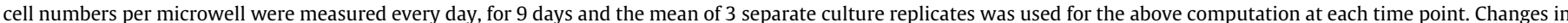

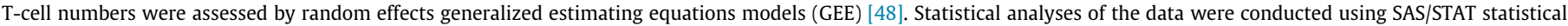
software version 9.1 (SAS Institute Inc., Cary, NC) and Stata 8.2 (StataCorp, College Station, TX). (For more detailed methods and additional results see [41]).

\begin{tabular}{|c|c|c|c|c|}
\hline \multirow[t]{2}{*}{ Variable } & \multicolumn{2}{|c|}{ CD4 T-cell subset } & \multicolumn{2}{|c|}{ CD8 T-cell subset } \\
\hline & Naïve $27+R A+$ & Memory 27-RA+/- \& 27+RA- & Naïve $27+R A+$ & Memory 27-RA+1- \& 27+RA- \\
\hline HIV serostatus (reference HIV-) & ${ }^{\mathrm{a}} 0.21(.30)$ & $0.10(.32)$ & ${ }^{\mathrm{a}} 0.12(.44)$ & $0.53(.52)$ \\
\hline Time (per day) & $-0.21(.03)^{* *}$ & $-0.37(.06)^{* * *}$ & $-0.33(.03)^{* *}$ & $-0.21(.03)^{* * *}$ \\
\hline Time $^{2}\left(\right.$ per day $\left.{ }^{2}\right)$ & - & $0.02(.01)^{* *}$ & - & - \\
\hline Virion treatment (X4 vs. reference R5) & $-0.09(.04)^{*}$ & $0.04(.04)$ & $0.02(.05)$ & $-0.04(.04)$ \\
\hline
\end{tabular}

a The unit of coefficients are cells/ $\mu \mathrm{l}$. (SE - standard error).

${ }^{*} p<0.01$.

$p<0.001$. 
able for 4 years on ART prior to the study. After the first ART interruption, viral load became detectable within 2 weeks to a set point of 39,000 copies/ml of plasma RNA. The patient was then put back on the ART therapy for approximately 5 months with the addition of UDL IL-2. IL-2 therapy was continued for 1.5 months after a second ART interruption. Five months after the second interruption (3.5 months after IL-2 therapy ceased), the patient's viral load was only 4 copies/ml of plasma RNA and the patient remained clinically undetectable for HIV for a total of 14 months off all therapy [56].

Unfortunately, none of these studies have resulted in the universal acceptance or application of these treatments because results vary considerably among different patients. It is still not clear how best to administer the therapies. It is not clear what immune profile distinguishes patients who are good candidates for and responders to each particular treatment [57]. It is also unclear when to intervene with these treatments and how to monitor and evaluate their success. These factors will become easier to determine once biomarkers for HIV disease progression and immune decline, in addition to CD4 T-cell levels and viral load, have been characterized more precisely and become widely acceptable.

Based on the hypothesis described in this article, additional biomarkers that could be useful for defining immune status in HIV infected individuals and that could help in developing and evaluating immune-restorative and cure-related treatments include markers for T-cell activation and death; markers for "partial" T-cell activation and immune exhaustion; peripheral IL-2 levels; naïve CD4 and CD8 T-cell levels; overall CD8 T-cell levels and T-cell function, including the proliferative capacity of naïve T-cell subsets, the cytotoxic capacity of CD8 T-cells and the ability of CD4 and central memory CD8 T-cells to secrete IL-2 and other cytokines and chemokines that are important for their function.

Despite the great strides in anti-retroviral therapy, it is important that we continue to study and develop immune-restorative therapies. This can be exemplified by the first case of a sterilization cure from HIV. A German HIV positive patient with acute myeloid leukaemia received a bone marrow transplant from an HIV-negative donor homozygous for a deletion in the CCR5 gene in 2009. The patient ceased HIV treatment soon after the procedure. There was a reconstitution and great improvement in the patient's immune profile. He currently remains undetectable for HIV nucleic acids and asymptomatic for HIV-related disease. This is especially encouraging considering that the patient did harbor X4 virus that his immune system was able to contain and eradicate after the immune-reconstructive therapy [58-60]. Elite controllers of HIV infection, who manage to keep viral loads below the limit of detection despite not being on any treatment, also point towards the possibility of a "functional cure" from HIV if researchers can determine how to boost the patient's own immune system [61]. Therapies that augment current anti-retroviral treatments are needed because generally, patients experience a viral rebound if they are taken off ART, even when their viral loads have become undetectable using ultra-sensitive molecular assays [62,63]. Moreover, long-term ART therapy is not readily available worldwide and is often unsustainable and prohibitively expensive to the local people in areas of the world that are the most affected by the epidemic.

\section{Conflict of interest}

There are no conflicts of interest to declare.

\section{Acknowledgments}

We thank Dr. Jeff Lifson, National Cancer institute, Frederick, $\mathrm{MD}$, for providing inactivated virions and the protocols for their use. We thank Elizabeth K. Cahoon for statistical assistance. We thank Gene Cimino and Paul Fallon for their technical assistance. We thank Akila Hadji, Stacey Meyerer and Shivam Chandan for their facilitation of blood donations. We thank Drs. Marie Hardwick, Alan Scott, Janice Clements, Pratip Chattopadhyay, Wen Chi and Yingbei Chen for helpful discussions.

\section{References}

[1] Adleman LM, Wofsy D. Blind T-cell homeostasis in CD4-deficient mice. J Acquir Immune Defic Syndr Hum Retrovirol 1996;11:334-40.

[2] Adleman LM, Wofsy D. T-cell homeostasis: implications in HIV infection. ] Acquir Immune Defic Syndr 1993;6:144-52.

[3] Fevrier M, Dorgham K, Rebollo A. CD4+ T cell depletion in human immunodeficiency virus (HIV) infection: role of apoptosis. Viruses 2011;3:586-612.

[4] Galai N, Margolick JB, Astemborski J, Vlahov D. Existence and failure of T-cell homeostasis prior to AIDS onset in HIV-infected injection drug users. Clin Immunol Immunopathol 1996;79:134-41.

[5] Gange SJ, Munoz A, Chmiel JS, et al. Identification of inflections in T-cell counts among HIV-1-infected individuals and relationship with progression to clinical AIDS. Proc Natl Acad Sci USA 1998:95:10848-53.

[6] Maas JJ, Gange SJ, Schuitemaker H, Coutinho RA, van Leeuwen R, Margolick JB. Strong association between failure of $\mathrm{T}$ cell homeostasis and the syncytiuminducing phenotype among HIV-1-infected men in the Amsterdam Cohort Study. AIDS 2000:14:1155-61.

[7] Margolick JB, Donnenberg AD, Munoz A, et al. Changes in $T$ and nonlymphocyte subsets following seroconversion to HIV-1: stable CD3+ and declining $C D 3$ - populations suggest regulatory responses linked to loss of CD4 lymphocytes. The Multicenter AIDS Cohort Study. J Acquir Immune Defic Syndr 1993;6:153-61.

[8] Margolick JB, Munoz A, Donnenberg AD, et al. Failure of T-cell homeostasis preceding AIDS in HIV-1 infection. The Multicenter AIDS Cohort Study. Nat Med 1995; $1: 674-80$

[9] Koot M, Keet IP, Vos AH, et al. Prognostic value of HIV-1 syncytium-inducing phenotype for rate of CD4+ cell depletion and progression to AIDS. Ann Intern Med 1993;118:681-8.

[10] Shepherd JC, Jacobson LP, Qiao W, et al. Emergence and persistence of CXCR4tropic HIV-1 in a population of men from the multicenter AIDS cohort study. J Infect Dis 2008:198:1104-12.

[11] Waters L, Mandalia S, Randell P, Wildfire A, Gazzard B, Moyle G. The impact of HIV tropism on decreases in CD4 cell count, clinical progression, and subsequent response to a first antiretroviral therapy regimen. Clin Infect Dis 2008;46:1617-23.

[12] Piatak Jr M, Saag MS, Yang LC, et al. High levels of HIV-1 in plasma during all stages of infection determined by competitive PCR. Science 1993;259:1749-54.

[13] Rossio JL, Esser MT, Suryanarayana K, et al. Inactivation of human immunodeficiency virus type 1 infectivity with preservation of conformational and functional integrity of virion surface proteins. J Virol 1998;72:7992-8001.

[14] Roederer M, Dubs JG, Anderson MT, Raju PA, Herzenberg LA, Herzenberg LA. CD8 naive T cell counts decrease progressively in HIV-infected adults. J Clin Invest 1995;95:2061-6.

[15] Gulzar N, Diker B, Mihowich J, et al. Proportion of HIV-1 infected CD8+CD4- T lymphocytes in vivo. Curr HIV Res 2008;6:585-96.

[16] McBreen S, Imlach S, Shirafuji T, et al. Infection of the CD45RA+ (naive) subset of peripheral CD8+ lymphocytes by human immunodeficiency virus type 1 in vivo. J Virol 2001;75:4091-102.

[17] Badley AD, Pilon AA, Landay A, Lynch DH. Mechanisms of HIV-associated lymphocyte apoptosis. Blood 2000;96:2951-64.

[18] Carbonari M, Pesce AM, Cibati M, et al. Death of bystander cells by a novel pathway involving early mitochondrial damage in human immunodeficiency virus-related lymphadenopathy. Blood 1997:90:209-16.

[19] Che KF, Sabado RL, Shankar EM, et al. HIV-1 impairs in vitro priming of naive T cells and gives rise to contact-dependent suppressor T cells. Eur J Immunol 2010;40:2248-58.

[20] Doitsh G, Cavrois M, Lassen KG, et al. Abortive HIV infection mediates CD4 T cell depletion and inflammation in human lymphoid tissue. Cell 2010;143:789-801.

[21] Espert L, Varbanov M, Robert-Hebmann V, et al. Differential role of autophagy in CD4 T cells and macrophages during X4 and R5 HIV-1 infection. PLoS One 2009; $4:$ e5787

[22] Finkel TH, Tudor-Williams G, Banda NK, et al. Apoptosis occurs predominantly in bystander cells and not in productively infected cells of HIV- and SIVinfected lymph nodes. Nat Med 1995;1:129-34.

[23] Ahr B, Robert-Hebmann V, Devaux C, Biard-Piechaczyk M. Apoptosis of uninfected cells induced by HIV envelope glycoproteins. Retrovirology $2004 ; 1: 12$.

[24] Garg H, Blumenthal R. Role of HIV Gp41 mediated fusion/hemifusion in bystander apoptosis. Cell Mol Life Sci 2008;65:3134-44.

[25] Molina L, Grimaldi M, Robert-Hebmann V, et al. Proteomic analysis of the cellular responses induced in uninfected immune cells by cell-expressed X4 HIV-1 envelope. Proteomics 2007;7:3116-30. 
[26] Smith DS, Guo K, Barrett BS, et al. Noninfectious retrovirus particles drive the APOBEC3/Rfv3 dependent neutralizing antibody response. PLoS Pathog 2011;7:e1002284.

[27] Chattopadhyay PK, Douek DC, Gange SJ, Chadwick KR, Hellerstein M, Margolick JB. Longitudinal assessment of de novo $T$ cell production in relation to HIV-associated $\mathrm{T}$ cell homeostasis failure. AIDS Res Hum Retroviruses 2006;22:501-7.

[28] Esser MT, Bess Jr JW, Suryanarayana K, et al. Partial activation and induction of apoptosis in CD4(+) and CD8(+) T lymphocytes by conformationally authentic noninfectious human immunodeficiency virus type $1 . \mathrm{J}$ Virol 2001;75:1152-64.

[29] Arthur LO, Bess Jr JW Chertova EN, et al. Chemical inactivation of retrovira infectivity by targeting nucleocapsid protein zinc fingers: a candidate SIV vaccine. AIDS Res Hum Retroviruses 1998;14(Suppl. 3):S311-9.

[30] Keet IP, Krol A, Koot M, et al. Predictors of disease progression in HIV-infected homosexual men with CD4+ cells < $200 \times 10(6) / 1$ but free of AIDS-defining clinical disease. AIDS 1994;8:1577-83.

[31] Gange SJ, Lau B, Phair J, Riddler SA, Detels R, Margolick JB. Rapid declines in total lymphocyte count and hemoglobin in HIV infection begin at CD4 lymphocyte counts that justify antiretroviral therapy. AIDS 2003;17:119-21.

[32] Levy JA. Virus-host interactions in HIV pathogenesis: directions for therapy. Adv Dent Res 2011;23:13-8.

[33] Chalmet K, Dauwe K, Foquet L, et al. Presence of CXCR4-using HIV-1 in patients with recently diagnosed infection: correlates and evidence for transmission. J Infect Dis 2012;205:174-84.

[34] Yang Y, Al-Mozaini M, Buzon MJ, et al. CD4 T-cell regeneration in HIV-1 elite controllers. AIDS 2012;26:701-6.

[35] Genesca M. Characterization of an effective CTL response against HIV and SIV infections. J Biomed Biotechnol 2011;2011:103924.

[36] Walsh AD, Bimber BN, Das A, et al. Acute phase CD8+ T lymphocytes against alternate reading frame epitopes select for rapid viral escape during SIV infection. PLoS One 2013;8:e61383.

[37] Schmitz JE, Kuroda MJ, Santra S, et al. Control of viremia in simian immunodeficiency virus infection by CD8+ lymphocytes. Science 1999;283:857-60.

[38] Walker UA, Warnatz K. Idiopathic CD4 lymphocytopenia. Curr Opin Rheumatol 2006;18:389-95.

[39] McDermott AB, Koup RA. CD8(+) T cells in preventing HIV infection and disease. AIDS 2012;26:1281-92.

[40] Eichbaum Q. PD-1 signaling in HIV and chronic viral infection - potential for therapeutic intervention? Curr Med Chem 2011;18:3971-80.

[41] Catherine Kibirige JM. Non-infectious CXCR4-tropic HIV induces T-Cell death and possibly AIDS. Dudweiler Landstr. 99, 66123 Saarbucken, Germany: Lambert Academic Publishing AG \& Co. KG; 2010.

[42] Choudhary SK, Walker RM, Powell DM, Planelles V, Walsh C, Camerini D. CXCR4 tropic human immunodeficiency virus type 1 induces an apoptotic cascade in immature infected thymocytes that resembles thymocyte negative selection. Virology 2006;352:268-84.

[43] Kitchen SG, Zack JA. CXCR4 expression during lymphopoiesis: implications for human immunodeficiency virus type 1 infection of the thymus. J Viro 1997;71:6928-34.

[44] Yang LP, Riley JL, Carroll RG, et al. Productive infection of neonatal CD8+ T lymphocytes by HIV-1. J Exp Med 1998;187:1139-44.
[45] Kitchen SG, Korin YD, Roth MD, Landay A, Zack JA. Costimulation of naive CD8(+) lymphocytes induces CD4 expression and allows human immunodeficiency virus type 1 infection. J Virol 1998;72:9054-60.

[46] Hoffman TL, LaBranche CC, Zhang W, et al. Stable exposure of the coreceptorbinding site in a CD4-independent HIV-1 envelope protein. Proc Natl Acad Sci USA 1999;96:6359-64.

[47] Kaslow RA, Ostrow DG, Detels R, Phair JP, Polk BF, Rinaldo Jr CR. The Multicenter AIDS Cohort Study: rationale, organization, and selected characteristics of the participants. Am J Epidemiol 1987;126:310-8.

[48] Carriere I, Bouyer J. Choosing marginal or random-effects models for longitudinal binary responses: application to self-reported disability among older persons. BMC Med Res Methodol 2002;2:15.

[49] Gupta SB, Jacobson LP, Margolick JB, et al. Estimating the benefit of an HIV-1 vaccine that reduces viral load set point. J Infect Dis 2007;195: 546-50.

[50] Noraz N, Gozlan J, Corbeil J, Brunner T, Spector SA. HIV-induced apoptosis of activated primary CD4+ T lymphocytes is not mediated by Fas-Fas ligand. AIDS 1997;11:1671-80.

[51] Kishore M, Ma L, Cornish G, Nourshargh S, Marelli-Berg FM. Primed T cell responses to chemokines are regulated by the immunoglobulin-like molecule CD31. PLoS One 2012;7:e39433.

[52] Chitnis V, Pahwa R, Pahwa S. Determinants of HIV-specific CD8 T-cell responses in HIV-infected pediatric patients and enhancement of HIVgag-specific responses with exogenous IL-15. Clin Immunol 2003;107: 36-45.

[53] Herbein G, Mahlknecht U, Batliwalla F, et al. Apoptosis of CD8+ T cells is mediated by macrophages through interaction of HIV gp120 with chemokine receptor CXCR4. Nature 1998;395:189-94.

[54] Okazaki T, Honjo T. PD-1 and PD-1 ligands: from discovery to clinical application. Int Immunol 2007;19:813-24.

[55] Denizot M, Varbanov M, Espert L, et al. HIV-1 gp41 fusogenic function triggers autophagy in uninfected cells. Autophagy 2008;4:998-1008.

[56] Margolick JB, Imteyaz H, Gallant JE, et al. Prolonged viral suppression without therapy in an HIV-1 seroconverter following early antiretroviral therapy and daily interleukin-2. AIDS 2010;24:932-5.

[57] Butler SL, Valdez H, Westby M, et al. Disease-modifying therapeutic concepts for HIV in the era of highly active antiretroviral therapy. J Acquir Immune Defic Syndr 2011;58:297-303.

[58] Lewin SR, Rouzioux C. HIV cure and eradication: how will we get from the laboratory to effective clinical trials? AIDS 2011;25:885-97.

[59] Hutter G, Schneider T, Thiel E. Transplantation of selected or transgenic blood stem cells - a future treatment for HIV/AIDS? J Int AIDS Soc 2009;12 (10-265212-10).

[60] Hutter G, Nowak D, Mossner M, et al. Long-term control of HIV by CCR5 Delta32/Delta32 stem-cell transplantation. N Engl J Med 2009;360:692-8.

[61] Mudd PA, Martins MA, Ericsen AJ, et al. Vaccine-induced CD8+ T cells control AIDS virus replication. Nature 2012:491:129-33.

[62] Chun TW, Justement JS, Murray D, et al. Rebound of plasma viremia following cessation of antiretroviral therapy despite profoundly low levels of HIV reservoir: implications for eradication. AIDS 2010;24:2803-8.

[63] Rasmussen TA, Schmeltz Sogaard O, Brinkmann C, et al. Comparison of HDAC inhibitors in clinical development: Effect on HIV production in latently infected cells and T-cell activation. Hum Vaccine Immunother 2013;9. 